Aus dem Anatomischen Institut der Med. Fakultät Okayama (Vorstaud : Prof. M. SEKI).

\title{
Die submikroskopische Strukturdichte der Linse und der benachbarten faserigen Gewebe des Menschen und ihre Altersveränderungen.
}

人の水晶体之近接線維組織の次微視的構密度とその年路的變化.

Von

\author{
Yasuharu HARADA. 原田保治.
}

(Eingegangen am 10. Febr. 1950.)

Die Gewebselemente des tierischen Körpers sind bekanntlich von verschiedener Sţrukturdichte. Schon früher hat unter anderen PAPPENHEIM (1901) darauf aufmerksam gemacht, daß ein Farbstoff vom höherer Dispersität ein dicht strukturiertes Gewebe färben kann, wenn es einem grobdispersen Farbstoff nicht gelingt, es zu färben. Die Theorie von MÖLLENDORFF (1924) und SEKI $(1932,1939)$ hat dazu Anwendung gefunden, die submikroskopische strukturdichte von Gewebselementen durch die kombinierte Verwendung von \%wei oder drei sauren Farbstoffen von verschiedener Dispersität und Farbe auf zufinden. Bei der HEIDENHAINschen Azanfärbung färben sich gewisse weitporige Gewebselemente, z. B. die Kollagenfasern, mit dem großmolekularen Anilinblau (Atomzahlen 78) blau und die Engporigen, z. B. die Muskelfasern, mit dem kleinmolekularen Azokarmin (Atomzahlen 62) rot, aber die extrem dicht strukturierten Gebilde mit dem gelben Goldorange G (Atomzahlen 35 ?) gelb. Das Zytoplasma und diesem ähnliche Gewebselemente nehmen einen gemischten Ton an.

Die vorliegende Arbeit ist vorgenommen, um festzustellen, wie die Strukturdichte der Linse und der benachbarten Fasergewebe, d.h. des ZINNschen Bändchens und der Glaskörpermembran, sich besonders mit zunehmendem Alter verändert. Die Linse spielt wie bekannt bei Durchgang und Brechung des lichtes eine große Rolle. SUGITA (1936) färbte die Linse des Rindes, der Ratte und des Frosches mit einem Farbstoff, Oxaminblau 4R, welcher von OHARA (1932) bei der Färbung der Seidenfäden angewandt wurde. Aus dem Resultate der Seidenfärbung muß man entnehmen, daß dieser Farbstoff ein saurer ist und hauptsächlich aus großmolekularen blauen Molekülen besteht, zu dem als Nebenbestandteile eine kleine Menge von mittelmolekularen roten und kleinmolekularen gelben vorhanden ist. Die Färbung mit. Oxaminblau 4R erfolgt also wie bei der Azanmethode. 


\section{Material und Methode.}

Als Material dienten uns normale Augen aus den Lejchen von 4 und 6 Fötalmonaten, Neugeborenen, 13, 41, 60 und 65 Jahren. Das Auge des 60 jährigen wurde gleich nach dem Tode genommen, die übrigen Augen aber aus mit Formalin in die Blutgefäße injizierten, in Formalinlösung aufbewahrten Leichen. Nachdem ich den vorderen halben Teil des Augapfels in Formalin weiter fixiert und in Zelloidin eingebettet hatte, verfertigte ich die Meridionalschnitte von $20 \mu$. Dicke. Man färbte die Schnitte nach der HEIDENHAINschen Methode, welche für die Entscheid ung der Strukturdichte am besten geeignet ist, Die Zelloidin färbte sich dabei ein wenig, doch wurde meine Beobachtung dadurch nicht gehindert. Die Konzentration der Farblösungen und die Zeitdauer der Färbung wurde verschieden abgeändert, aber entsprechend dem $Z_{w e c k}$ meiner Forschung, färbte ich eine Reihe Präparate, welche untereinander zu vergleichen waren, gleichzeitig. wusch sie auch gleichzeitig und fertigte sie um die gleiche Zeit an. Naçhdem ich aber die Tatsache bestätigt hatte, daß die Linse aus dem Embryo, Neugeborenen und 60 jährigen der Reihe nach dichter wird, teilte ich das ganze Material in \%wei Teile, der erste vom 4. Fötalmonat bis zum 13. Lebensjahr, der zweite vom 13._65. Lebensjahr. Um die folgenden Ergebnisse zu erzielen, färbte man die Schnitte mit Azokarmin B 30 bis 35 Minuten (bei $40^{\circ} \mathrm{C}$ ), brachte diese durch Anilinalkohol (ein Minut), in die Phosphorwolframsäurelösung ein (eine Stunde) und färbte sie 2.5 bis 3 Minuten in einer zweifach verdünnten Anilinblau-Orange G Lösung, welche noch einen kleinen Zusatz von Orange $\mathrm{G}$ erhalten hatte und gelbbraun aussah.

\section{Untersuchungsergebnisse.}

\section{A. Linsensubstanz.}

In allen Alterstufen lassen sich die Linsenfasern konzentrisch von der Oberfläche der Linse nach innen hin getrennt in der Ordnung von Blau, Rosa und Rotgelb färben. Der blaue Teil gilt der Rinde, der Rosateil der $Z_{w}$ ischenschicht und der rotgelbe Teil dem Kern. Mikroskopisch scheinen auch die Fasern des blauen Teils sich locker zu ordnen, und die der anderen zwei Farbenteile im Gegenteil verdichtet zu sein. Die Strukturdichte der Linse ist also submikroskopisch wie mikroskopisch : Rinde $>Z_{\text {wischenschicht }}>$ Kern.

Im 4 und 6 Fötalmonat (Fig. 1-2) ist der blaue Teil größer als der rotgelbe, und zwar ist der blaue Teil in der Vorderseite schmal, in der Gegend des Äquators verbreitet und ist in der Hinterseite wieder schmal. Der blaue Teil ist im vorderen Pol am schmalsten, und die 
Grenze zwischen Blau und Rosa ist dort nicht deutlich. Beim Neugeborenen (Fig. 3) ist das Verhältnis ungefähr mit dem des Embryos gleich, aber der rotgelbe Teil hat sich ein wenig vergrößert. Beim 41jährigen (Fig. 5) ist der rotgelbe Teil weitaus vergrößert, und der blaue Teil bleibt sehr schmal in der vorderen und hinteren Seite und auch im Aquator. Die rosa $Z_{w}$ ischenschicht ist am schmalsten, Die Grenze zwischen Blau und Rosa ist deutlicher. Beim Mann über 60 Jahre alt (Fig. 6-7) nimmt der rotgelb färbbare Teil den größten Teil der Linse ein, und der sehr schmal gewordene blaue Randteil nimmt nunmehr einen violetten Farbenton an, indem er nur sichelförmig vom Äquator bis zum vorderen und hinteren Teil liegen bleibt. Genauer untersuch1, ist in jedem Alter dicht unter dem Epithel fast immer ein enges rotes Strukturgebiet zu finden. Im Alter von mehr als 41 Jahren ist dicht unter der hinteren Kapsel auch eine ganz schmale rote Schicht bemerkbar.

Aus oben erwähnten 'latsachen geht hervor, daß die dichten Kernfasern nach und nach auf Kosten der lockeren Randschicht den Hauptteil der Linse einnehmen. In diesem Zusammenhange wollte ich den Altersveränderungen der Strukıurdichte des Linsenkerns selbst näher kommen und modifizierte die Färbungswege verschiedenartig, aber ich konnte keine regelmäßigen Resultate bekommen. Die Strukturdichte des Linsenkerns scheint merkwürdigerweise immer fast gleich zu bleiben.

\section{B. Linsenkapsel.}

Im 4. Fötalmonat färbt sich die Linsenkapsel blau, nur daß ihre Innerseite eine violette Nuance zeigt. Im 6. Fötalmonat wird sie blauviolett, beim Neugeborenen violett, im 13. Jahre violett oder ein wenig rosaviolett, im 41 . Jahre violett oder violettrosa, und danach violettrosa färbbar. Sie ist unter dem Mikroskop immer strukturlos. Die Reihenfolge der Strukturdichte der Linsenkapsel ist in ; 4. Fötalmonat $\leqq 6$. Fötalmonat $<1$. Monat $\leqq 13$. Jahr $\leqq 41$. Jahr $=60$. Jahr $\leqq 65$. Jahr. Vergleicht man die Kapseln vom Embryo, Neugeborenen und 13 jährigen unter dem Mikroskop, so ist das Dickerwerden derselben augenscheinlich. Auch ein kleiner Unterschied zwiscben dem 13- und 41- und mehr jährigen ist bemerkbar.

\section{ZINNsche Bändchen.}

Die Strahlenbändchenfasern sind beim Embryo und Neugeborenen blaßblau, beim 13 jährigen violettblau bis blauviolett, beim 41 jährigen violettblau bis violett und beim 60-65 jährigen bläulichrot bis rosarot. Die Strukturdichte des Strahlenbändchens nimmt also mit 
dem Alter zu. Besonders zwischen dem Neugeborenen und den alten Leuten läßt sich ein beträchtlicher Unterschied bemerken. Beim Embryo sind übrigens die Bändchenfasern ganz dünn, beim Neugeborenen sind sie ein wenig dicker. Nach dem 13. Jahre werden sie weitaus dicker.

\section{Glaskörpermembran.}

Beim Embryo ist die vordere Grenzschicht des Glaskörpers gan\% dünn und blaßblau färbbar, beim Neugeborenen wird sie ein wenig dicker und bildet eine Faserschicht. Die Fasern werden dann mit einer mit Violett angehauchten blaßblauen Farbe gefärbt. Im 13. Jahr nimmt die Grenzmembran in Dicke und in blauer Färbbarkeit $z u$, zeigt aber danach keine regelmäßigen Veränderungen. Kurz, von der Embryonalzeit bis in hohem Alter ist eine beträchtliche Zunahme der strukturdich te nicht wahrzunehmen.

\section{Schlußbetrachtung.}

1. Linse.

Die Linse läßt das Licht durchgehen, brechen und wirft ein Bild auf die Netzhaut. Deshalb muß sie vollständig klar sein. Ist die Dichte der Linsensubstanz gleichmäßig? Nach den oben erwähnten Untersnchung ist die submikroskopische Dichte dieser durchsichtigen Substanz keineswegs gleichmäßig. Es ließ sich färberisch die T'atsache beweisen, daß die Linse aus einer lockeren äußeren Schicht, einer dichteren $Z$ wischenschicht und einem dichtesten Kern besteht. SUGITA (1936) färbte die tierische Linsen mit Oxaminblau $4 \mathrm{R}$ und hatte analoge Befunde, aber die Linse in verschiedenen Altersstufen wurden dabei nicht näherer Betrachtung gezogen. Im allgemeinen ist die Substanz von dichterer Struktur optisch auch dichter und besitzt einen höheren Brechungsindex. In der Tat ist ophthalmophysiologisch bestätigt, da $\beta$ der Brechungsindex der verschiedenen Teile der Linse ist: Rinde $<Z_{W}$ ischenschicht $<$ Kern, eine Reihe, die mit derjenigen der Strukturdichte übereinstimmt.

Die Strukturdichte der Linse verändert sich ferner mit zunehmendem Alter. Der aus den dicht strukturierten Fasern bestandene Kernteil vergrößert sich von der Embryonalzeit bis ins hohe Alter, so daß der Kerntejl schließlich den Hauptteil der Linse einnimmt. Demgegenüber wird der Rindenteil aus lockeren Fasern immer dünner. Bekanntlich verlängern sich die Epithelzellen am Äquator der Linse, und die so entstandenen Linsenfasern fügen sich an die Oberfläche zu. Deshalb vergrößert sich der Durchmesser und die Dicke der Linse bis zum hohen Alter. Die aus dem Epithel neu gebildeten Fasern sind also locker strukturiert und werden mit der Zeit immer dichter. Nach 
SUGITA soll dagegen ein im Äquatorbezirk von tierischen Linsen befindlicher halbmondförmiger Teil dicht strukturiert sein. Beim Menschen befindet sich, wie oben erwähnt, eine dichte strukturierte Schicht unter dem vorderen Epithel, in späteren Lebensallern aber auch unter der hinteren Kapsel.

Im Jugendalter ist die Linse leicht deformierbar, reich an Elastizität und hat eine große Akkommodationsbreite, verliert aber mit dem Altern ihre Elastizität und wird von mehr flacher Form. Es ist also anzunehmen, daß die Verminderung der weniger dichten Rindenfasern mit der Sklerose der Linse Hand in Hand geht. Die Tatsache, daß in der Jugendzeit die weniger dichten Rindenfasern vom Äquator bis im hinteren Teil reichlich vorhanden sind, scheint eine Beziehung mil der stärkeren Krümmung der hinteren Linsenoberfläche zu haben. Die weitere Tatsache, daß bei fortschreitendem Alter sowohl in der vorderen als auch in der hinteren oberflächlichen Schicht eine dünne, wenig dichte Rindensubstanz bestehen bleibt, steht vielleicht damit in Zusammenhang, daß beim Alten die Krümmung an den beiden Flächen ungefähr gleich ist.

Die Linsenkapsel nimmt, wenn auch nicht stark, an Dichte zu. Sie wird auch dicker. Die Verdickung wurde schon von SALZNANN (1912) u.a. gemessen. Die Vermehrung der Dichte und Dicke der Linsenkapsel hat meines Erachtens zu der bekannten Tatsache Beziehung, daß der Brechungsindex und die Elastizität der Kapsel mit dem Altern zunimmt. Nach SUGITA (1036) ist die Linsenkapsel mit Farbstoffen nicht färbbar, so daß sie am dichtesten gebaut ist. Diese Meinung ist vielleicht das Ergebnis einer ungünstigen Färbungsmethode. Die Linsenkapsel des Menschen färbt sich, wie erwähnt, ganz deutlich, und zwar ist die Dichte nie am. größten und verändert sich mit dem Altern.

Was die Strukturdichte verschiedener Teile der Linse in jeder Lebenszeit betrifft, so ist sie in der Embryonalzeit : Rinde $\leqq$ Kapsel $<$ Zwischenschicht $<$ Kern, nach der Geburt : Rinde $<$ Kapsel $<$ Zwischenschicht $<$ Kern und im hohen Alter : Rinde $\leqq$ Kapsel $\leqq Z_{w i-}$ schenschicht $<$ Kern.

\section{ZINNsches Bändchen.}

Eine histologische Untersuchung über das ZINNsche Bändchen wurde in Japan neuerdings von SHIMOYAMA (1937) an menschlichen Augen vorgenommen. Über die Strukturdichte desselben ist aber keine Beschreibung vorhanden. Als BEAUVIEUX (1922) das Menschenauge mit Anilinolau und Orange $G$ färbte, bemerkte er, daB die Linse sich gelb und das Strahlenbändchen sich schön blau färbte, aber er hatte keine Ahnung von deren Dichte. Nach meiner Untersuchung 
sind die Bändchenfasern beim Embryös dünn und wenig dicht strukturiert, und werden beim Neugeborenen ein wenig dicker, aber nicht dichter. Nach 13 Jahren entwickeln sich die Fasern gut und werden noch etwas dicker. Ihre Strukturdichte erhöht sich erst beim Alten beträchtlich. V. PFLUGK (1932) fand, daß die Dehnbarkeit der Bändchenfasern sehr groß ist, wie die der Linsenkapsel. CALDERARO (1917) fand ferner, daB der Widerstand gegen die Ausdehnung bei den Jungen viel stärker ist als beim Alten. Die Strukturdichte der Bändchenfasern ist, wie oben erwähnt, bei den Jungen weniger dicht als bei den Alten.

3. Vordere Grenzschicht des Glaskörpers.

Das Vorhandensein der Glaskörpermembran ist seit früher viel umstritten. In den fixierten mikroskopischen Präparaten erscheinen aber die Fasern in dem peripheren Teil des Glaskörpers zusammengedrängt und bilden an der vorderen Grenze gegen die Hinterkammer eine Membran, die vordere Grenzschicht. Diese verdickt sich mit der Entwickelung und vermehrt gleichzeitig ihre Strukturdichte, wie ich oben erwähnt habe.

4. Vergleichende Beobachtung der Strukturdichte der Linsenkapsel, des ZINNschen B̈̈ndchens und der Vordergrenzschicht des Glaskörpers.

Im 4. bis 6. Fötalmonat ist die Strukturdichte : Linsenkapsel $\geqq$ ZINNsches Bändchen = vordere Grenzschicht des Glaskörpers. Nach der Geburt : Kapsel $>$ ZINNsches Bändchen = vordere Grenzschicht. Bei hohem Alter : ZINNsches Bändchen $>$ Kapsel $>$ vordere Grenzschicht, das ZINNsche Bändchen ist dabei am dichtesten.

\section{Zusammenfassung.}

An sieben Augen im Alter von 4 Fötalmonaten bis zu 65 Jahren wurde die submikroskopische Strukturdichte der Linse, des ZINNschen Bändchens und der vorderen Grenzschicht der Glaskörpermembran auf färberischem Wege untersucht, und zwar nach der Azanmethode.

1. Die Strukturdichte des Linsenkerns ist immer höher als diejenige der Rinde. Mit zunehmendem Alter vergrößert sich der dichte Kernteil auf Kosten der weniger dichten Rinde.

2. Die Linsenkapsel nimmt sehr langsam an Dichte $z u$.

3. Die Dichte des ZINNschen Bändchens nimmt auch allmählich zu, um dann in hohem Alter sich noch schneller zu verdichten. Das Bändchen wird endlich etwas dichter als die Linsenkapsel. Es bleibt aber, immer weniger dicht als der Linsenkern. 
4. Die vordere Grenzschicht des Glaskörpers ist immer locker strukturiert. Genauer gesagt wird sie aber von der Embryonalzeit bis zum mittlerem Alter etwas dichter.

\section{內 容自 抄}

胎生 4 月から 65 歲に至る人の水晶體, Zinn 氏小帶, 硝子體膜の次微視 的䩢密度を Heidenhain 氏アツァン染色法により檢查した. 水晶體中央の 棈密度は常に皮質部のものより高く, 年路と其に密構の核部は垻大し, 反 對に柾構の皮質は狹くなる・水昆體被膜は楛だ徐々に棈密度を增すＺinn 氏小帶の構密度は徐々に增し, 老年に至つて速かに貹して水晶體被膜のも のより高くなるが，常に水晶體侅の構密度よりは低い. 硝子體前境界屓は 常に柾構である.

\section{Literatur.}

Beauvieux : Ia zonule. Arch. p'Ophthalm. 39 (1922). - Calderaro : 1917. zit. n. Lauber (Möllendorffs Handbuch, Bd. 3). - Möllendorff, W. u. M. v. : Untersuchungen zur Theorie der Färbung fixierter Präparate. III. Ergebn. Anat. 25 (1924). - Ỏhara, K. : Erforschung des feinen Baues der Seiden. II. Rikagaku-Kenkyusho-Iho. 11 (1932). - Pappenheim, A. : Grundriß der Farbchemie. Berlin. 1901. - Pflu k, A. v. : Neue Wege zur Erforschung der Lehre von der Akkommodation. Graefes A rch. 128 (1932). - Salzmann : 1912. zit. n. Lauber (Möllendorffs Haudbuch Bd. 3). - Seki, M. : Zur physikalischen Chemie der histologischen Färbung. II. Fol. anat. jap. 10 (1932). - Untersuchungen mit nichtwässerigen Flüssigkeiten. VIII. Z. Zellforsch. 29 (1939). - Sugita, Y. : Kolloidchemische Betrachtungen über die verschiedenen Teile des Auges speziell über dem Unterschied »wischen der Hellnetzhaut und Dunkelnet\%haut. Chuo-Ganka Tho. 28 (1936), - Shimoyama, T. : Über die Zonula Zinni. Acta Soc. ophthalm. jap. 41, VI. Suppl. (1937).

\section{Erklärung der Tafelabbildungen.}

Abb. 1. 4. Linse im Fötalmonat.

Abb. 3. Linse des Nengeborenen.

Abb. 5. Im 41. Jahre.

Abb. 7. Im 65. Jah re.
Abb. 2. Im 6. Fötalmonat.

Abb. 4. Linse in 13. Jahre.

Abb. 6. Im 60. Jahre. 
原田保治論文附圖

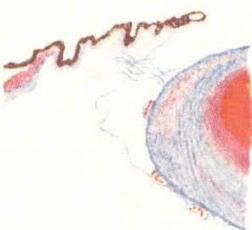

1. 4. Fötalmonat.

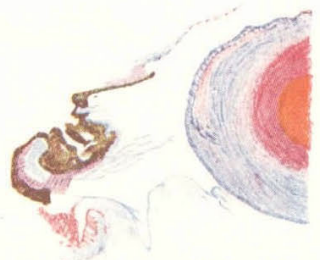

2. 6. Fötalmonat.

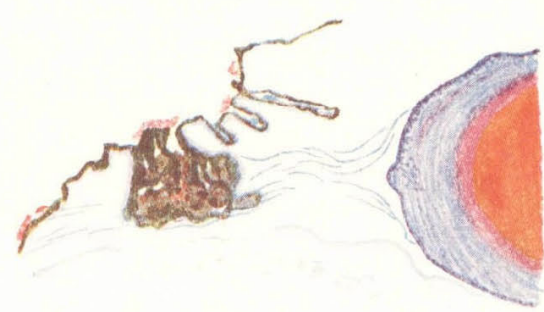

3. Neugcborenes.

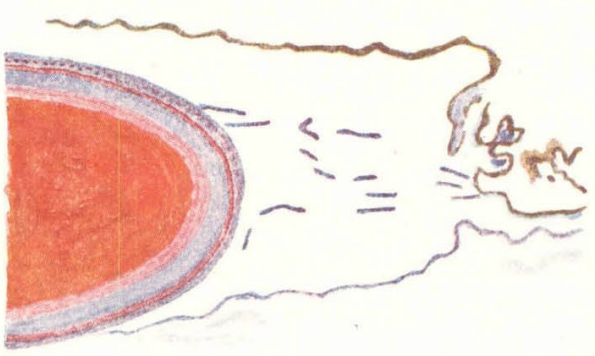

5. 41. Jahr.

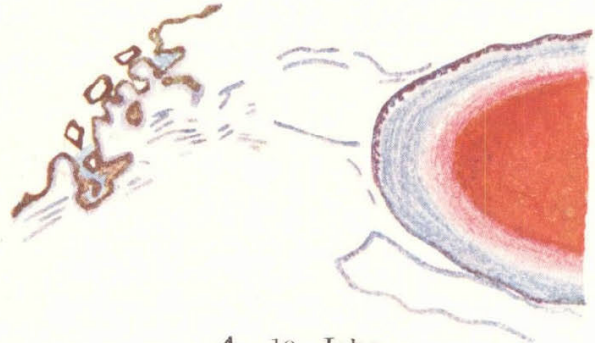

4. 13. Jahr.

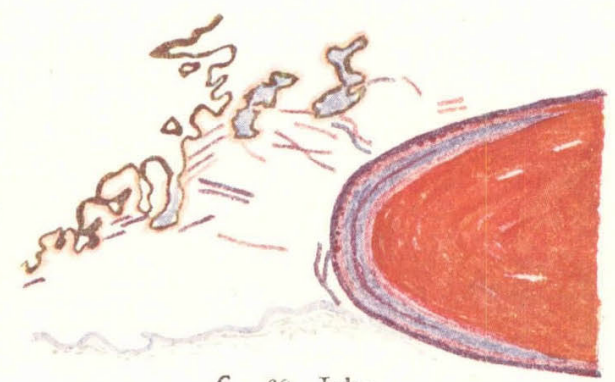

6. 60. Jahr

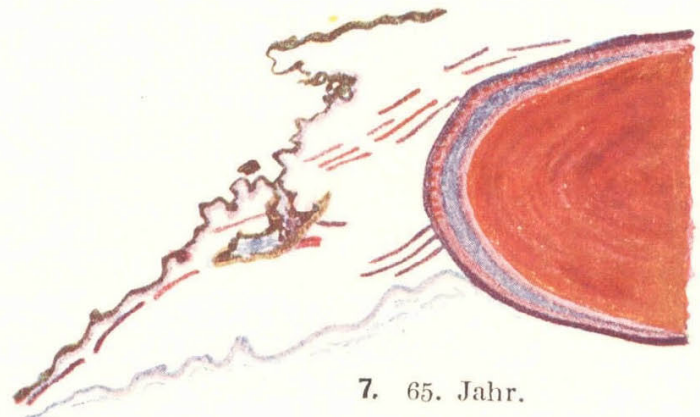

